\title{
Clinical characterisation and risk stratification of patients with arrhythmogenic right ventricular dysplasia/cardiomyopathy $\geq 50$ years of age
}

\author{
M. J. van der Pols ${ }^{1}$ T. P. Mast ${ }^{1}$ P. Loh Lo. F. van der Heijden $^{1}$ - M. J. Cramer ${ }^{1}$ R. N. Hauer ${ }^{3}$ A. S. J. M. te \\ Riele ${ }^{1,2,3}$
}

Published online: 31 August 2016

(C) The Author(s) 2016. This article is available at SpringerLink with Open Access.

\begin{abstract}
Purpose With the increased use of genetic testing for arrhythmogenic right ventricular dysplasia/cardiomyopathy $(\mathrm{ARVD} / \mathrm{C})$, this disease is being increasingly recognised among elderly patients. However, elderly ARVD/C patients were underrepresented in prior cohorts. We aimed to describe the phenotypical characteristics and outcomes among ARVD/C patients surviving $\geq 50$ years.

Methods We assessed detailed phenotypical data of 29 patients who (1) presented at $\geq 50$ years of age; and (2) fulfilled 2010 Task Force Criteria (TFC) for ARVD/C by last follow-up. Primary outcome was the occurrence of a major ventricular arrhythmia (sudden cardiac death, resuscitated sudden cardiac arrest or sustained ventricular tachycardia). Results The majority (55\%) of elderly ARVD/C subjects were male, with a mean age of $59.0 \pm 5.8$ years at presentation. Study participants fulfilled a median of six (IQR 5-8) TFC criteria by last follow-up, of which arrhythmia criteria were most frequent $(97 \%)$, followed by structural criteria $(83 \%)$, depolarisation criteria $(72 \%)$ and repolarisation criteria (69\%). By last follow-up, $15(52 \%)$ patients had experienced major ventricular arrhythmias. Most patients $(n=12)$ presented with this arrhythmia, while three experienced the event during $5.4 \pm 3.2$ years of followup. Compared with patients without an arrhythmic event, patients with major arrhythmias were more likely to be
\end{abstract}

\footnotetext{
A. S. J. M. te Riele

ariele3@umcutrecht.nl

1 Department of Cardiology, University Medical Center Utrecht, Utrecht, The Netherlands

2 Department of Cardiology, Johns Hopkins University School of Medicine, Baltimore, MD, USA

3 Netherlands Heart Institute, Utrecht, The Netherlands
}

proband $(p<0.001)$ and male $(p=0.042)$. Likewise, survival free from sustained ventricular arrhythmia was lower among probands and males.

Conclusion Phenotypic characteristics of elderly ARVD/C patients are characterised by depolarisation abnormalities and structural cardiac changes. Ventricular arrhythmias in this elderly cohort are associated with male gender and proband status.

Keywords Arrhythmogenic right ventricular dysplasia . Cardiomyopathy · Elderly · Ventricular arrhythmia .

Clinical phenotype

\section{Introduction}

Arrhythmogenic right ventricular dysplasia/cardiomyopathy (ARVD/C) is an inherited cardiomyopathy characterised by fibrofatty replacement of predominantly the right ventricular myocardium, frequent ventricular arrhythmias, and an increased risk of sudden cardiac death [1,2]. ARVD/C patients classically present between the second and fifth decade of life with symptomatic ventricular arrhythmias [3]. However, with increased use of molecular genetic testing for ARVD/C, the disease is more frequently recognised among elderly patients. Objective data describing the phenotypic characteristics and clinical course of elderly ARVD/C patients are currently lacking, which complicates recommendations for the management of this growing group of patients.

The objectives of this study were twofold. First, we aimed to describe the phenotypical characteristics and clinical course in a cohort of patients presenting with ARVD/C at the age of 50 years or older. Second, we sought to identify predictors of sustained ventricular arrhythmias that 
would be of value in determining which ARVD/C patients would benefit from more intensive screening or prophylactic implantation of an implantable cardioverter-defibrillator.

\section{Methods}

\section{Study population}

The study population was recruited from the University Medical Center Utrecht ARVD/C registry [4]. We identified 29 patients enrolled in the registry who (1) presented for first clinical evaluation at $\geq 50$ years, and (2) were diagnosed with definite ARVD/C in accordance with the 2010 Task
Force Criteria (TFC) by last follow-up [5]. Ascertainment of the study population is shown in Fig. 1.

\section{Clinical evaluation}

Patients were evaluated as described previously [6]. Mutation analysis of the desmosomal genes encoding plakophilin-2 (PKP2), desmoplakin (DSP), desmoglein-2 (DSG2), desmocollin-2 (DSC2), and plakoglobin (JUP), and the non-desmosomal gene phospholamban $(P L N)$ was performed in all patients, as reported previously [6, 7]. Criteria for pathogenicity of variants were determined as done previously [4]. Twelve-lead electrocardiograms (ECGs) (recorded at rest, $10 \mathrm{~mm} / \mathrm{mV}$ at paper speed $25 \mathrm{~mm} / \mathrm{s}$ ) were
Fig. 1 Ascertainment of the study population (UMCU University Medical Center Utrecht)

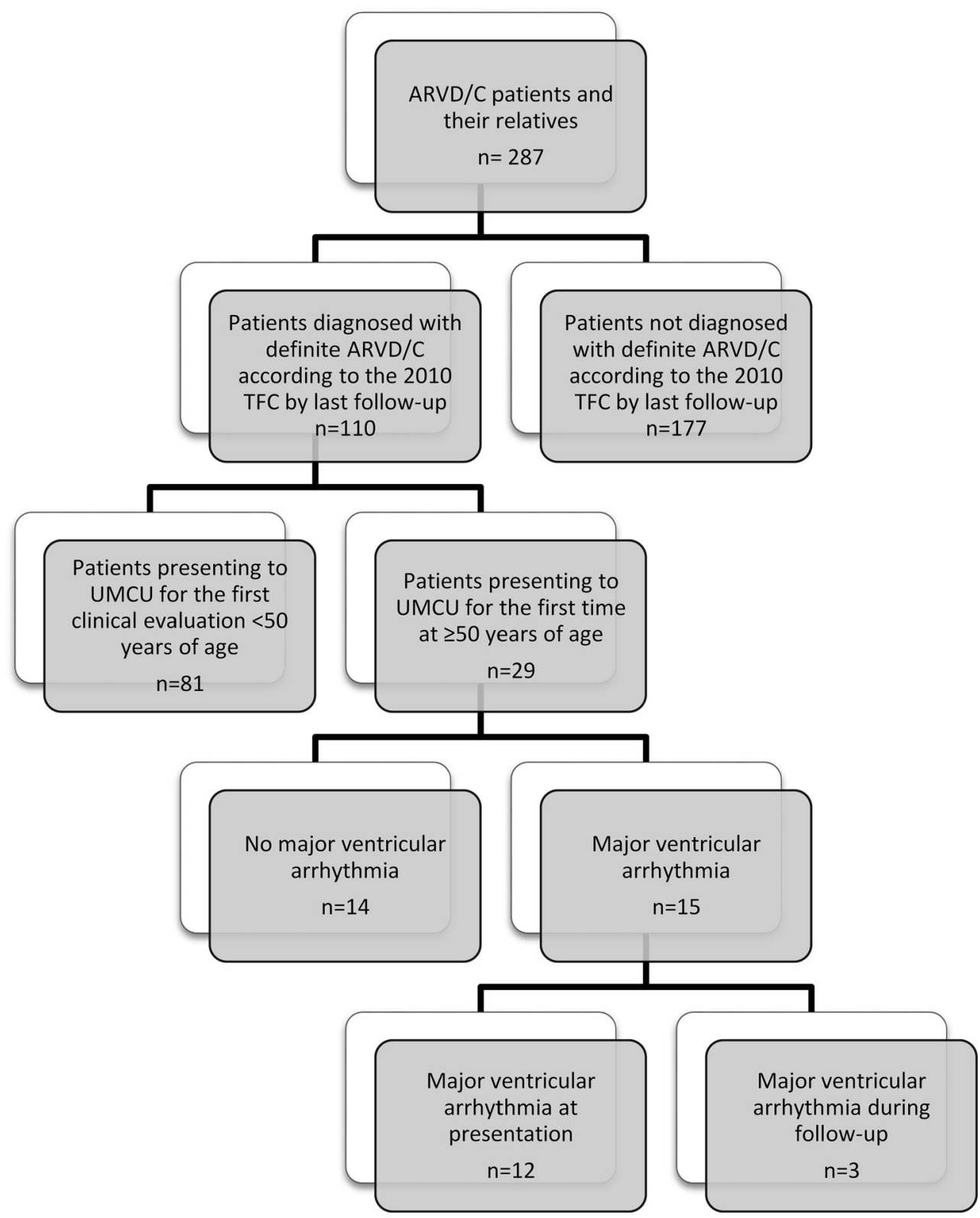


evaluated for repolarisation (precordial T-wave inversion in V1-2 or beyond) and/or depolarisation (epsilon waves or terminal activation duration $\geq 55 \mathrm{~ms}$ ) criteria for ARVD/C. All patients were off drugs that influence ventricular depolarisation or repolarisation at the time of ECG recording. Twenty-four hour Holter monitoring was used to determine the frequency of premature ventricular complexes (PVC). Exercise stress testing was performed to detect ventricular tachycardia (VT) and ischaemia. Since signal-averaged ECG was not available in this cohort, we used invasive electrophysiological study as a measure of late potentials. Electrophysiological studies were performed in 15 subjects, the majority of whom $(11 / 15,73 \%)$ had prior VT, while the remainder $(4 / 15,27 \%)$ were symptomatic and had frequent ectopy on Holter monitoring. Late potentials on electrophysiological study were defined as local electrogram signals after termination of the QRS complex on the surface ECG during sinus rhythm. Echocardiography, cardiac magnetic resonance (CMR) imaging, and right ventricular angiography were reviewed to determine cardiac structural abnormalities. Per study design, all study participants fulfilled diagnostic criteria for definite ARVD/C according to the revised 2010 TFC [5].

\section{Outcome}

The primary outcome measure was the occurrence of a major ventricular arrhythmia, which was a composite measure of the occurrence of sudden cardiac death (SCD), resuscitated sudden cardiac arrest (SCA), or spontaneous sustained VT ( $\geq 100 /$ min, lasting $\geq 30 \mathrm{~s}$ or shorter with electrical or pharmacological interruption), as done previously [8]. In subjects with multiple endpoints, the first event was considered to be the censoring event.

\section{Statistical analysis}

Continuous data are displayed as mean \pm standard deviation or median (interquartile range [IQR]) and as proportions for categorical variables. Differences in continuous data were calculated using the independent samples $t$-test or Mann-Whitney $U$ test as appropriate; for categorical data the Chi-square test or Fisher's exact test was used. KaplanMeier curves were calculated for time to an event of a major ventricular arrhythmia. $P$-values $<0.05$ were considered significant. Data were analysed using SPSS software (version 22.0 for Mac).

\section{Results}

\section{Study population}

We included 29 patients who presented at $\geq 50$ years of age and were diagnosed with ARVD/C by last follow-up. Their phenotypical characteristics are summarised in Table 1 . Overall, $16(55 \%)$ study participants were male, with a mean age of $59.0 \pm 5.8$ years at the time of presentation. As shown in Fig. 2, the majority $(n=15,52 \%)$ of patients presented between the age of 50 and 60 years. ARVD/C-associated pathogenic mutations were found in 21 (72\%) patients; mutations were most frequently observed in the plakophilin-2 $(n=14,48 \%)$ gene, while the remainder harboured a phospholamban mutation $(n=7,24 \%)$.

Table 1 Phenotypic characteristics of the study population

\begin{tabular}{|c|c|}
\hline & $\begin{array}{l}\text { Definite ARVD/C }(n= \\
\text { 29) }\end{array}$ \\
\hline \multicolumn{2}{|l|}{ Demographics } \\
\hline Male & $16(55)$ \\
\hline Age at presentation (years) & $59.0 \pm 5.8$ \\
\hline Proband & $16(55)$ \\
\hline Mutation carrier & $21(72)$ \\
\hline Plakophilin-2 & $14(48)$ \\
\hline Phospholamban & $7(24)$ \\
\hline \multicolumn{2}{|l|}{ Clinical phenotype at last follow-up } \\
\hline Age at diagnosis (years) & $59.9 \pm 6.7$ \\
\hline Repolarisation TFC & $20(69)$ \\
\hline T-wave inversion V1-3 & $16(55)$ \\
\hline T-wave inversion V1-2 & $2(7)$ \\
\hline T-wave inversion V4-6 & $2(7)$ \\
\hline $\begin{array}{l}\text { T-wave inversion V1-4 with complete } \\
\text { RBBB }\end{array}$ & $0(0)$ \\
\hline Depolarisation TFC & $21(72)$ \\
\hline Epsilon wave & $5(17)$ \\
\hline$>\mathrm{TAD}$ & $17(59)$ \\
\hline Late potentials & $8 / 18(44)$ \\
\hline Arrhythmia TFC & $28(97)$ \\
\hline Holter abnormal & $16 / 21(76)$ \\
\hline PVC count; median [IQR] & $2056[560-5952]$ \\
\hline LBBB superior axis VT & $13(45)$ \\
\hline LBBB VT & $15(52)$ \\
\hline Structural TFC & $24(83)$ \\
\hline Major & $21(73)$ \\
\hline Minor & $3(10)$ \\
\hline TFC points; median [IQR] & $6[5-8]$ \\
\hline
\end{tabular}

$A R V D / C$ Arrhythmogenic right ventricular dysplasia/cardiomyopathy, $R B B B$ right bundle branch block, $I Q R$ interquartile range, $L B B B$ left bundle branch block, $P V C$ premature ventricular complex, $>T A D$ prolonged terminal activation duration, TFC Task Force Criteria, $V T$ ventricular tachycardia 


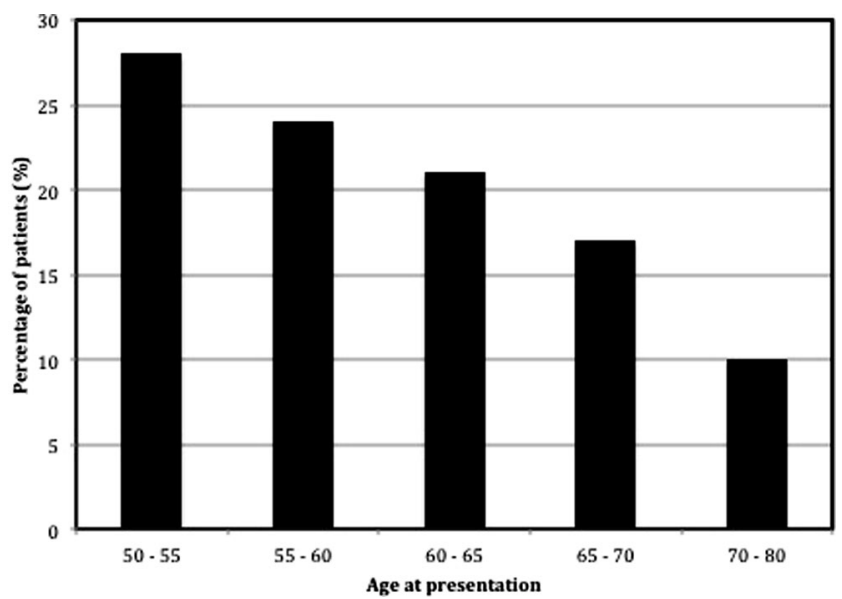

Fig. 2 Age distribution of the study population. The distribution of age at first clinical cardiac evaluation among the study population. Overall, $28 \%$ presented at $50-55$ years, $24 \%$ at 55-60 years, $21 \%$ at $60-65$ years, $17 \%$ at $65-70$ years and $10 \%$ at $70-80$ years of age. Mean age at time of presentation was $59.0 \pm 5.8$ (range 50.2-77.9) years

\section{Clinical evaluation}

The majority ( $n=23,79 \%)$ of patients fulfilled the diagnostic TFC at the time of first presentation; the remainder $(n=6,21 \%)$ were diagnosed on average $3.6 \pm 2.2$ years after presentation. Reasons for clinical presentation were sustained monomorphic VT in $12(41 \%)$ patients, family screening in $10(34 \%)$ patients, and syncope/presyncope or palpitations in $7(24 \%)$ patients. No study subjects presented with an SCD or resuscitated SCA.

As shown in Table 1, T-wave inversions were observed in $20(69 \%)$ elderly patients, most commonly ( $n=16$, $55 \%$ ) in leads V1-3. Depolarisation abnormalities were observed in $21(72 \%)$ patients, of which terminal activation duration $\geq 55 \mathrm{~ms}$ was the most frequent $(n=17,59 \%)$. Twenty-four hour Holter monitoring showed $>500$ PVCs in $16 / 21$ subjects $(76 \%)$. The majority of patients $(n=24$, $83 \%$ ) had structural abnormalities on imaging studies. The median TFC score was 6 (IQR 5-8) at last follow-up.

\section{Ventricular arrhythmia}

Among the overall population, 15 (52\%) patients experienced a major ventricular arrhythmia, either at first presentation $(n=12,80 \%)$ or during follow-up $(n=3,20 \%)$. All first major ventricular arrhythmias presented with spontaneous sustained VT. Clinical characteristics of these patients are shown in Table 2. Compared with subjects without arrhythmia, patients who experienced an arrhythmic event were more likely to be male $(n=11[73 \%]$ vs. $n=$ $5[36 \%] ; p=0.042)$ and proband $(n=13[87 \%]$ vs. $n=3$ [21\%]; $p<0.001)$. There were no other differences in de-

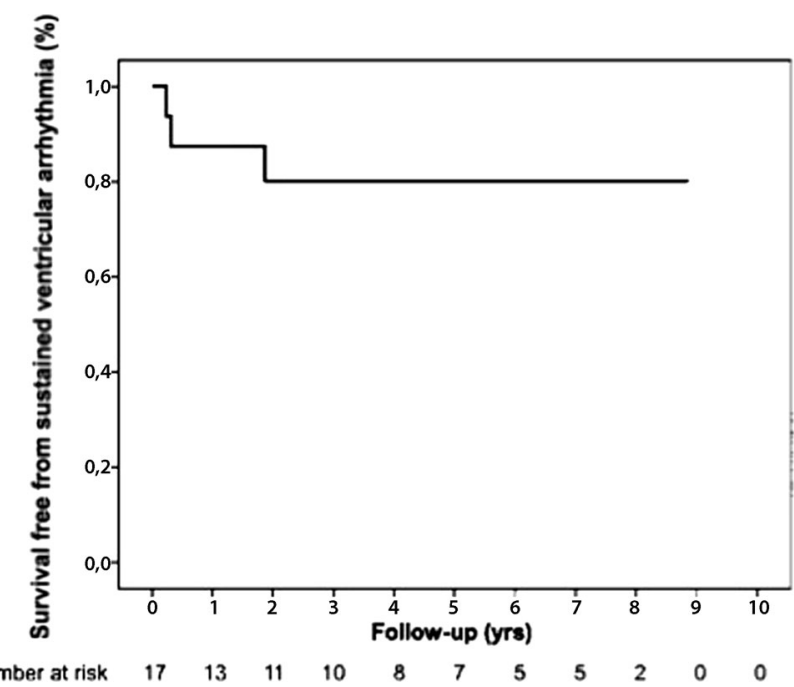

Fig. 3 Survival free from major ventricular arrhythmias among the study population. Kaplan-Meier survival analyses demonstrating time to first major ventricular arrhythmia during follow-up among the overall cohort

mographics or in any domain of the TFC between subjects with and without arrhythmia.

In elderly patients, an obvious arrhythmic substrate may occur in the setting of coronary artery disease (CAD). Electrocardiographic evidence of previous myocardial infarction was absent in all patients. In addition, we evaluated the presence or absence of CAD using coronary angiography and exercise stress testing in 15 patients with ventricular arrhythmia. Two $(13 \%)$ of these patients had evidence of CAD, defined as $>50 \%$ stenosis on coronary angiography. The first subject presented at age 77 with left bundle branch block (LBBB) inferior axis VT, and was found to have a $70 \%$ stenosis in the circumflex artery on coronary angiography. CMR revealed right ventricular abnormalities suggestive of ARVD/C, and further evaluation revealed T-wave inversions in V1-4 and a radical mutation in the plakophilin-2 gene (deletion exon 1-14). The second subject presented at 76 years of age with VT of LBBB superior axis morphology and a normal left ventricular ejection fraction of $54 \%$. However, a $70 \%$ stenosis in margo obtusus 1 was found and subsequently treated with a bare metal stent. The LBBB superior axis VT recurred, after which another percutaneous coronary intervention was performed. After several years of recurrent VT, a comprehensive follow-up revealed ARVD/C based on late potentials, major structural CMR abnormalities, and LBBB superior axis VT. All other patients had no evidence of CAD on coronary angiography $(n=11)$ and/or exercise stress testing $(n=14)$. 
Table 2 Phenotypic characteristics of the study population

\begin{tabular}{|c|c|c|c|}
\hline & $\begin{array}{l}\mathrm{VT} \\
(n=15)\end{array}$ & $\begin{array}{l}\text { No VT } \\
(n=14)\end{array}$ & $p$ value \\
\hline \multicolumn{4}{|l|}{ Demographics } \\
\hline Male & $11(73)$ & $5(36)$ & 0.042 \\
\hline Proband & $13(87)$ & $3(21)$ & $<0.001$ \\
\hline Mutation carrier & $10(67)$ & $11(79)$ & 0.682 \\
\hline Plakophilin-2 & $7(47)$ & $7(50)$ & 0.862 \\
\hline Phospholamban & $3(20)$ & $4(29)$ & 0.682 \\
\hline \multicolumn{4}{|l|}{ Mode of presentation } \\
\hline Family screening & $0(0)$ & $10(71)$ & $<0.001$ \\
\hline Symptomatic & $3(20)$ & $4(29)$ & 0.682 \\
\hline Sustained VT & $12(80)$ & $0(0)$ & $<0.001$ \\
\hline SCA & $0(0)$ & $0(0)$ & - \\
\hline SCD & $0(0)$ & $0(0)$ & - \\
\hline \multicolumn{4}{|l|}{ Clinical phenotype at last follow-up } \\
\hline Age at diagnosis (years) & $62.5 \pm 9.7$ & $60.9 \pm 6.4$ & 0.623 \\
\hline T-wave inversion V1-3 & $7(47)$ & $9(64)$ & 0.340 \\
\hline T-wave inversion V1-2 & $2(13)$ & $0(0)$ & 0.483 \\
\hline T-wave inversion V4-6 & $0(0)$ & $2(14)$ & 0.224 \\
\hline T-wave inversion V1-4 with complete RBBB & $0(0)$ & $0(0)$ & - \\
\hline Epsilon wave & $4(27)$ & $1(7)$ & 0.330 \\
\hline$>\mathrm{TAD}$ & $9(60)$ & $8(57)$ & 0.876 \\
\hline Late potentials & $5 / 11(45)$ & $3 / 7(43)$ & 1.000 \\
\hline Holter monitor abnormal & $5 / 8(63)$ & $11 / 13(85)$ & 0.325 \\
\hline PVC count; median [IQR] & 919 [341-2095] & 3108 [1205-6141] & 0.205 \\
\hline Major structural TFC & $13(87)$ & $8(57)$ & 0.109 \\
\hline Minor structural TFC & $1(7)$ & $2(14)$ & 0.598 \\
\hline TFC points; median [IQR] & $6[5-7]$ & $6[5-8]$ & 0.506 \\
\hline
\end{tabular}

$R B B B$ right bundle branch block, $I Q R$ interquartile range, $P V C$ premature ventricular complex, $S C A$ sudden cardiac arrest, $S C D$ sudden cardiac death, $>T A D$ prolonged terminal activation duration, $T F C$ Task Force Criteria, $V T$ ventricular tachycardia

\section{Ventricular arrhythmia during follow-up}

Fig. 3 shows cumulative survival free from major ventricular arrhythmia in 17 patients who did not present with an arrhythmia. Over a mean follow-up of $5.4 \pm 3.2$ years, $3 / 17(18 \%)$ patients experienced a sustained monomorphic VT. Median cycle length of the VT was $240 \mathrm{~ms}$ (range 231-429). VT episodes showed LBBB morphology: two with superior, one with inferior axis. As shown in Fig. 3, median time to first VT was 4 months after first cardiological evaluation. Cumulative survival free from major ventricular arrhythmia after 6, 12 and 24 months was $88 \%$ (95\% CI 72.3-100.0), $88 \%$ (95\% CI 72.3-100.0), and $80 \%$ (95\% CI 60.4-99.6), respectively.

The characteristics of the three patients who experienced an arrhythmic event during follow-up are shown in Table 3. The first event was experienced at a median age of 59 (range 55-69) years. Interestingly, all patients with arrhythmia were male and proband, and one of them was a carrier of an ARVD/C-associated pathogenic mutation (plakophilin-2). Prior to the arrhythmia, all three patients had re- ported symptoms including exercise-induced syncope ( $n=$ $2)$, palpitations $(n=2)$, and/or presyncope $(n=1)$. At the time of the arrhythmia, all patients fulfilled the TFC for ARVD/C: repolarisation criteria were fulfilled in $1 \mathrm{pa}-$ tient (major), depolarisation criteria in 2 patients ( 1 major, 1 minor), and arrhythmia criteria in 3 patients (2 major, 1 minor) (Table 3). In addition, cardiac imaging showed that all 3 subjects had major structural abnormalities. Distribution of age at diagnosis $(p=0.885)$, sex $(p=0.516)$ and presence of a pathogenic mutation $(p=0.242)$ was similar in patients with arrhythmic events at presentation and during follow-up.

\section{Discussion}

This study has several interesting results. First, we found that the clinical phenotype of elderly ARVD/C patients is characterised by depolarisation abnormalities on 12-lead ECG, frequent PVCs on Holter monitoring, and structural alterations. Molecular genetic testing revealed a high per- 
Table 3 Characteristics of three patients experiencing first VT episode during follow-up

\begin{tabular}{|c|c|c|c|}
\hline & Patient \#1 & Patient \#2 & Patient \#3 \\
\hline \multicolumn{4}{|l|}{$\overline{\text { Demographics }}$} \\
\hline Sex & M & M & M \\
\hline Age at time of diagnosis (years) & 55 & 69 & 59 \\
\hline Proband & Yes & Yes & Yes \\
\hline Pathogenic mutation & - & - & $+(P K P 2)$ \\
\hline \multicolumn{4}{|l|}{ Sustained arrhythmia characteristics } \\
\hline Age at time of arrhythmia & 55 & 71 & 59 \\
\hline Morphology & LBBB & LBBB & LBBB \\
\hline Axis & Superior & Superior & Inferior \\
\hline Cycle length (ms) & 240 & 231 & 429 \\
\hline Documentation & Walking & Cardiac stress test & Holter monitoring \\
\hline \multicolumn{4}{|c|}{ Clinical phenotype at time of sustained arrhythmia } \\
\hline Repolarisation TFC & None & None & TWI V ${ }_{1-4}$ (major) \\
\hline Depolarisation TFC & None & Late potentials (minor) & Epsilon wave (major) \\
\hline Arrhythmia TFC & $\begin{array}{l}\text { LBBB superior axis VT (ma- } \\
\text { jor) }\end{array}$ & $\begin{array}{l}560 \mathrm{PVCs} / 24 \mathrm{~h} \text { (minor), } \\
\text { LBBB superior axis VT } \\
\text { (major) }\end{array}$ & $\begin{array}{l}11180 \mathrm{PVCs} / 24 \mathrm{~h} \text { (minor), } \\
\text { LBBB inferior axis VT (mi- } \\
\text { nor) }\end{array}$ \\
\hline Structural TFC & $\begin{array}{l}\mathrm{RV} \text { aneurysm + reduced RV } \\
\text { function (major) }\end{array}$ & $\begin{array}{l}\text { RV aneurysm + reduced RV } \\
\text { function (major) }\end{array}$ & $\begin{array}{l}\text { RV aneurysm + reduced RV } \\
\text { function (major) }\end{array}$ \\
\hline Family history TFC & None & None & Major \\
\hline TFC points at time of arrhythmic event & 4 & 5 & 8 \\
\hline \multicolumn{4}{|l|}{ Clinical features } \\
\hline Symptoms prior to event & $\begin{array}{l}\text { Exercise-induced syncope, } \\
\text { presyncope }\end{array}$ & Palpitations & Palpitations \\
\hline Medication at time of arrhythmia & $\begin{array}{l}\text { Perindopril } 8 \mathrm{mg}, \mathrm{Hy}- \\
\text { drochlorothiazide } 25 \mathrm{mg}\end{array}$ & Atenolol $25 \mathrm{mg}$ & $\begin{array}{l}\text { Acenocoumarol }^{\mathrm{a}} \text {, Enalapril } \\
20 \mathrm{mg} \text {, Simvastatin } 40 \mathrm{mg}\end{array}$ \\
\hline Relevant comorbidity & Hypertension & - & Ischaemic CVA \\
\hline Coronary angiography & Normal & Normal & Normal \\
\hline
\end{tabular}

$C V A$ cerebrovascular accident, $L B B B$ left bundle branch block, $P K P 2$ Plakophilin-2, $P V C$ premature ventricular complex, $T F C$ Task Force Criteria, $T W I$ T-wave inversion, $V T$ ventricular tachycardia

${ }^{a}$ Indication for acenocoumarol was a history of ischaemic CVA

centage of phospholamban $p$.Arg14del mutation carriers. Second, risk of SCD was low in this elderly cohort, since none of the patients in this study experienced SCD/SCA. Last, arrhythmic events in this elderly cohort were associated with proband status and male gender, and all three patients experiencing events during follow-up were male and proband. These results provide interesting information for clinical practice, which is still largely based on expert opinion and consensus documents.

\section{Clinical phenotype in elderly patients}

Prior studies have extensively described phenotypical characteristics in ARVD/C patients [3, 7, 9]. As such, we know that depolarisation and repolarisation changes on 12-lead ECG and frequent ectopy on Holter monitoring are often observed in ARVD/C patients $[3,7,8]$. However, it is important to note that elderly patients were underrepresented in prior ARVD/C cohorts, which typically had a mean age between $20-40$ years $[3,7,9]$. To the best of our knowledge, the present study is the first to specifically focus on those ARVD/C patients who only come to clinical attention after the age of 50 years. As in younger ARVD/C subjects, ECG abnormalities, frequent ventricular ectopy, and structural alterations are commonly observed in this elderly cohort.

It is important to recognise that ventricular arrhythmias in the elderly population have a broad differential diagnosis and CAD, in particular, is much more prevalent than ARVD/C. Even in the 76-year-old patient in our ARVD/C cohort, monomorphic VT was initially thought to be related to the $70 \%$ stenosis in a coronary artery. However, monomorphic VT in CAD in the absence of a previous myocardial infarction is very rare. Although our study was not designed to compare the clinical characteristics of elderly subjects with and without ARVD/C, these results provide a cautionary note to cardiologists, who may wrongly disregard the diagnosis of ARVD/C based on older age at 
presentation. On the other hand, wall motion abnormalities of the right ventricle are common and cardiologists should be aware that non-pathological right ventricular wall motion disorders can easily be mistaken for a pathological regional wall motion contraction, particularly in patients with ARVD/C [10]. Future studies should address specific treatment recommendations with regard to coronary artery disease and its prevention among ARVD/C patients.

\section{Genotype of elderly ARVD/C subjects}

The yield of molecular genetic testing in ARVD/C typically lies between 50-63\% [7]. Our yield of 72\% is higher than in previously published reports. This may be due to a founder effect of the phospholamban $p$.Arg14del mutation [11], which was observed in a remarkably large subset of our population $(24 \%)$. Prior reports have shown that ARVD/C patients who harbour a phospholamban mutation present at an older age, frequently with left ventricular involvement [12, 13]. In this regard, it is interesting that only $62 \%$ of our population had T-wave inversion in the right precordial leads, in contrast to $>85 \%$ in the ARVD/C literature $[3,7,8]$. This may reflect a left predominant phenotype dominated by phospholamban mutations in our population.

\section{Elderly patients have a low risk of SCD}

Prevention of SCD is the most important therapeutic goal in ARVD/C [14]. Therefore, it is an important finding of our study that none of the elderly patients in our cohort experienced SCD/SCA either at presentation or during follow-up. This is in contrast to prior studies with young ARVD/C patients which reported a 3-22\% rate of SCD [3, 4, 7]. This finding is particularly interesting in the context of recent basic science studies showing that desmosomal mutations lead to reduced sodium current which promotes re-entry susceptibility at an early disease stage [15-17]. In contrast, heterogeneous fibrofatty replacement of the myocardium leads to monomorphic sustained VT that is haemodynamically stable and can be adequately treated. In our study population, $82 \%$ already had structural abnormalities on cardiac imaging. As such, we believe that our study subjects were more likely to experience haemodynamically welltolerated monomorphic VT, rather than ventricular fibrillation. Future studies are necessary to determine whether a true difference in arrhythmic substrate exists in younger vs. elderly ARVD/C subjects.

\section{Clinical characteristics in subjects with VT}

Despite a low risk of SCD, malignant ventricular arrhythmias are not uncommon among elderly ARVD/C patients.
Our study shows that more than half of ARVD/C patients who present beyond 50 years of age experience a ventricular arrhythmia during more than five years of follow-up. Almost all of these events occurred at presentation. As such, the risk of ventricular arrhythmias seems highest for as yet unrecognised cases. Ventricular arrhythmias were significantly associated with male gender and proband status, and arrhythmic events during follow-up occurred exclusively among male probands. These data suggest that further screening and invasive risk stratification in elderly ARVD/C subjects may be most beneficial among male probands.

\section{Study limitations}

This study was limited by its small sample size. However, this is consistent with a low prevalence of ARVD/C, possibly due to under-recognition. Since this is a registry-based study, not all subjects underwent all clinically available tests such as Holter monitoring. It is possible that Holter monitoring was mainly employed in those with mild disease, which may explain a relatively modest PVC count in this cohort. Data on exercise participation were not available. Given recent studies confirming the role of strenuous exercise in phenotypic development of ARVD/C [18, 19], it would be interesting to further investigate exercise participation in elderly ARVD/C subjects. Detailed analysis of the relationship between structural phenotype and age were beyond the scope of this study. Since a large proportion of elderly patients presented with ventricular arrhythmias, only cross-sectional analyses could be performed to determine predictors of arrhythmic events. While we are aware of the broad differential diagnosis of arrhythmias in an elderly population (particularly ischaemic heart disease), we were unable to obtain coronary angiography in all patients. Of note, all patients who did not undergo coronary angiography had no electrocardiographic (exercise stress testing or resting ECG) and CMR evidence of previous myocardial infarction.

\section{Conclusions}

The results of our study show that ARVD/C in elderly patients is characterised by depolarisation abnormalities, frequent ectopy on Holter monitoring, and structural changes on cardiac imaging, while molecular genetic analysis reveals a high percentage of carriers of the phospholamban p.Arg14del mutation. Ventricular arrhythmias are typically haemodynamically well-tolerated monomorphic VT and are associated with male sex and proband status. These results suggest that further evaluation and more aggressive risk stratification may be justifiable in male probands surviving beyond 50 years of age. Concomitant CAD remains 
a source of misinterpretation in the aetiology of monomorphic VT in this elderly age group.

Funding This work was supported by the Dutch Heart Foundation (2015T058 to A.S.J.M. te Riele) and by the Interuniversity Cardiology Institute of the Netherlands (ICIN), project 06901.

Conflict of interest M.J. van der Pols, T.P. Mast, P. Loh, J. F. van der Heijden, M. J. Cramer, R.N. Hauer and A.S.J.M. te Riele declare that they have no competing interests.

Open Access This article is distributed under the terms of the Creative Commons Attribution 4.0 International License (http:// creativecommons.org/licenses/by/4.0/), which permits unrestricted use, distribution, and reproduction in any medium, provided you give appropriate credit to the original author(s) and the source, provide a link to the Creative Commons license, and indicate if changes were made.

\section{References}

1. Marcus FI, Fontaine GH, Guiraudon G, et al. Right ventricular dysplasia: a report of 24 adult cases. Circulation. 1982;65:384-98.

2. Corrado D, Basso C, Thiene G, et al. Spectrum of clinicopathologic manifestations of arrhythmogenic right ventricular cardiomyopathy/dysplasia: a multicenter study. J Am Coll Cardiol. 1997;30:1512-20.

3. Dalal D, Nasir K, Bomma C, et al. Arrhythmogenic right ventricular dysplasia: a United States experience. Circulation. 2005; 112:3823-32.

4. Bhonsale A, Groeneweg JA, James CA, et al. Impact of genotype on clinical course in arrhythmogenic right ventricular dysplasia/cardiomyopathy-associated mutation carriers. Eur Heart J. 2015;36:847-55.

5. Marcus FI, McKenna WJ, Sherrill D, et al. Diagnosis of arrhythmogenic right ventricular cardiomyopathy/dysplasia: proposed modification of the task force criteria. Circulation. 2010;121:1533-41.

6. Cox MG, van der Zwaag PA, van der Werf C, et al. Arrhythmogenic right ventricular dysplasia/cardiomyopathy: pathogenic desmosome mutations in index-patients predict outcome of family screening: Dutch arrhythmogenic right ventricular dysplasia/ cardiomyopathy genotype-phenotype follow-up study. Circulation. 2011;123:2690-700.

7. Groeneweg JA, Bhonsale A, James CA, et al. Clinical presentation, long-term follow-up, and outcomes of 1001 arrhythmogenic right ventricular dysplasia/cardiomyopathy patients and family members. Circ Cardiovasc Genet. 2015;8:437-46.

8. Bhonsale A, James CA, Tichnell C, et al. Risk stratification in arrhythmogenic right ventricular dysplasia/cardiomyopathy-associated desmosomal mutation carriers. Circ Arrhythm Electrophysiol. 2013;6:569-78.

9. te Riele AS, James CA, Rastegar N, et al. Yield of serial evaluation in at-risk family members of patients with ARVD/C. J Am Coll Cardiol. 2014;64:293-301.

10. Quick S, Speiser U, Kury K, Schoen S, Ibrahim K, Strasser R. Evaluation and classification of right ventricular wall motion abnormalities in healthy subjects by 3-tesla cardiovascular magnetic resonance imaging. Neth Heart J. 2015;23:64-9.

11. van der Zwaag PA, van Rijsingen IA, de Ruiter R, et al. Recurrent and founder mutations in the netherlands-phospholamban p.Arg14del mutation causes arrhythmogenic cardiomyopathy. Neth Heart J. 2013;21:286-93.

12. Groeneweg JA, van der Zwaag PA, Olde Nordkamp LR, et al. Arrhythmogenic right ventricular dysplasia/cardiomyopathy according to revised 2010 task force criteria with inclusion of nondesmosomal phospholamban mutation carriers. Am J Cardiol. 2013;112:1197-206.

13. Groeneweg JA, van der Zwaag PA, Jongbloed JD, et al. Left-dominant arrhythmogenic cardiomyopathy in a large family: Associated desmosomal or nondesmosomal genotype? Heart Rhythm. 2013;10:548-59.

14. Groeneweg JA, van der Heijden JF, Dooijes D, van Veen TA, van Tintelen JP, Hauer RN. Arrhythmogenic cardiomyopathy: diagnosis, genetic background, and risk management. Neth Heart J. 2014;22:316-25.

15. Cerrone M, Noorman M, Lin X, et al. Sodium current deficit and arrhythmogenesis in a murine model of plakophilin-2 haploinsufficiency. Cardiovasc Res. 2012;95:460-8.

16. Rizzo S, Lodder EM, Verkerk AO, et al. Intercalated disc abnormalities, reduced na(+) current density, and conduction slowing in desmoglein-2 mutant mice prior to cardiomyopathic changes. Cardiovasc Res. 2012;95:409-18.

17. Delmar M, McKenna WJ. The cardiac desmosome and arrhythmogenic cardiomyopathies: from gene to disease. Circ Res. 2010;107:700-14.

18. James CA, Bhonsale A, Tichnell C, et al. Exercise increases agerelated penetrance and arrhythmic risk in arrhythmogenic right ventricular dysplasia/cardiomyopathy-associated desmosomal mutation carriers. J Am Coll Cardiol. 2013;62:1290-7.

19. te Riele AS, Marcus FI, James CA, et al. The value of cardiac magnetic resonance imaging in evaluation of pediatric patients for arrhythmogenic right ventricular dysplasia/cardiomyopathy. J Am Coll Cardiol. 2015;66:873-4. 